\title{
Les expulsés inexpulsables. Recompositions du contrôle des étrangers dans la France des années
} 1930

Nicolas Fischer

\section{(2) OpenEdition Journals}

Édition électronique

URL : http://journals.openedition.org/conflits/991

DOI : $10.4000 /$ conflits. 991

ISSN : $1777-5345$

Éditeur :

CCLS - Centre d'études sur les conflits lilberté et sécurité, L'Harmattan

Édition imprimée

Date de publication : 1 mars 2004

Pagination : 25-41

ISBN : 2-7475-6791-5

ISSN : 1157-996X

Référence électronique

Nicolas Fischer, « Les expulsés inexpulsables. Recompositions du contrôle des étrangers dans la

France des années $1930 »$, Cultures \& Conflits [En ligne], 53 | printemps 2004, mis en ligne le 04 octobre 2004, consulté le 30 mars 2021. URL : http://journals.openedition.org/conflits/991 ; DOI : https:// doi.org/10.4000/conflits.991

Ce document a été généré automatiquement le 30 mars 2021.

Creative Commons License 


\title{
Les expulsés inexpulsables. Recompositions du contrôle des étrangers dans la France des années 1930
}

\author{
Nicolas Fischer
}

1 Parmi les institutions mises en place au cours du XXème siècle pour l'exercice d'une surveillance politique, le camp a connu des usages très divers. Il paraît d'autant plus nécessaire de le réinsérer dans l'agencement général de la surveillance qui lui donne sens. Si le camp apparaît à un moment donné dans les pratiques matérielles et discursives, c'est que, comme l'indique Foucault à propos de la prison ${ }^{1}$, il a été sélectionné par le "diagramme », par la cartographie générale du pouvoir. Il faut donc penser l'insertion de l'institution dans un agencement plus général du pouvoir toujours fluctuant, instable et réversible, mais qui la relie à d'autres institutions, contemporaines ou antérieures, et aux pratiques discursives qui s'y connectent.

Dans ce qui suit, il s'agira d'analyser la création d'un dispositif général dont le camp ne constitue qu'une « pièce ». On parlera donc d'un type particulier de camp: les «Centres Spéciaux de Rassemblement » français, créés sous la IIIème République par le décret-loi du 12 novembre 1938, et dont un seul fut réellement mis en place à Rieucros, en Lozère, en janvier 1939. Mais ce type lui-même sera à distinguer en ce qu'il s'insère dans un ensemble de mesures - reconduite à la frontière, détention administrative - évoquées dès cette époque pour régler un problème très contemporain : celui de l'éloignement effectif des étrangers en instance d'expulsion. Il sera donc problématisé à la faveur d'un débat spécifiquement français sur la réforme de l'expulsion du territoire.

$\mathrm{Au}$ cours de la décennie 1930, la situation des quelques deux millions et demi d'étrangers que compte alors le pays est en effet fragilisée. Alors qu'ils sont pour une bonne partie d'entre eux déjà installés depuis plusieurs dizaines d'années, les effets de la crise économique, ressentis en France dès 1931, débouchent sur une augmentation des mesures d'éloignement du territoire. Face à la montée du chômage, elles 
concernent en premier lieu l'immigration étrangère de travail - Polonais, Italiens et Espagnols présents dans l'industrie et dans l'agriculture - dont on cherche à réduire le nombre ${ }^{2}$. Elles visent aussi l'afflux de réfugiés (Juifs allemands et opposants expulsés par les régimes autoritaires européens) provoqué par la radicalisation des conflits politiques internationaux à partir de $1933^{3}$.

4 Si toutes ces catégories sont visées par l'expulsion, c'est que la mesure n'est pas encadrée et qu'elle peut intervenir pour les motifs les plus divers ${ }^{4}$. Mais ce «flou », qui commence alors à poser problème, ne concerne pas que l'éloignement : il caractérise plus généralement la condition des étrangers en France. La réforme de l'expulsion est donc une partie d'un débat plus vaste sur le statut des étrangers qui marque la France des années $1930^{5}$. Elle se concentre notamment sur un enjeu: l'instauration d'un contrôle des étrangers, de plus en plus nombreux, auxquels on notifie effectivement un arrêté d'expulsion, mais qui demeurent malgré tout sur le territoire.

On voit donc naître un souci nouveau, celui «d'assurer le départ effectif des étrangers». Il s'articule autour de la mise en visibilité d'une nouvelle catégorie d'individus appelant une gestion différenciée, qui suppose elle-même la création d'instances spécialisées: c'est à cette occasion que la gestion des populations étrangères croisera la reconduite à la frontière et d'autres «innovations » - dont le camp - que l'Etat républicain institue à l'époque, ou se propose d'instituer.

6 Ces institutions spécifiquement dédiées à "l'effectuation matérielle de l'expulsion" vont se trouver problématisées dans des espaces particuliers, sociétés savantes ou clubs de réflexion héritiers des sociétés savantes criminologiques de la fin du XIXème siècle : c'est le cas du Congrès national des sociétés de patronage dans lequel est d'abord évoquée la réforme de l'expulsion ${ }^{6}$, mais aussi des travaux du Comité français de droit international privé 7 . Comme au siècle précédent, ces sociétés sont conçues par leurs membres comme des arènes de réflexion participant à la construction dans l'espace public des enjeux liés à la criminalité. Mais elles sont surtout envisagées comme des espaces d'expertise, participant et éventuellement déclenchant les réformes en la matière. Entretenant des liens plus ou moins formalisés mais durables avec les Assemblées, elles sont sensées «doubler» et préparer leur travail législatif, en constituant ainsi de véritables " commissions privées, extra-parlementaires » dont les travaux relayent l'intervention de la puissance publique ${ }^{8}$. Ce travail de problématisation passe également par la constitution autour de la question de "l'éloignement effectif " d'un réseau spécifique réunissant des acteurs d'origines diverses ${ }^{9}$. Les bulletins publiés par ces sociétés savantes en donnent une idée assez précise. Parmi les animateurs du débat sur les étrangers à l'époque, on y trouvera quelques « experts » en politiques d'immigration (Georges Mauco ou Marcel Paon ${ }^{10}$ ), et des juristes spécialistes du statut des étrangers, dont Jean-Paulin Niboyet ${ }^{11}$. S'y ajoutent cependant des acteurs appartenant plus directement au "champ pénal ", et qui à ce titre participent en priorité au débat concernant le traitement des criminels et l'économie de la répression ${ }^{12}$ - on y croise des magistrats ${ }^{13}$ et pénalistes reconnus, dont Paul Matter ${ }^{14}$. Dans les deux cas, théoriciens et praticiens du droit dominent, mais ils prennent place au sein d'un réseau particulier qui les relie aux acteurs administratifs de la réforme, élus, fonctionnaires ou " publicistes » qui seront invités à intervenir.

On n'évoquera pas dans ce qui suit la position individuelle de chaque acteur, mais bien plutôt les thèmes et les représentations qui leur sont communs sur la question de l'éloignement effectif : ils traduisent une recomposition partielle de l'organisation de la 
surveillance des étrangers. Autour d'une situation qui n'avait pas été auparavant constituée en problème, les acteurs qui s'expriment ici sont en effet tenus d'élaborer des techniques de contrôle spécifiquement destinées aux inexpulsables. Mais cette réorganisation, mêlant la réforme de l'expulsion et le débat sur l'économie du pénal et de l'enfermement, est « recadrée » dans le discours propre au champ pénal de l'époque, soit dans un ensemble de représentations héritées de la morale «disciplinaire » du XIXème siècle. C'est cette interaction entre des dispositifs nouveaux, et leur appréhension dans des termes hérités de la gestion des populations à surveiller du siècle précédent, que nous allons chercher à restituer dans ce qui suit.

"L'inexpulsable », problème nouveau ou illégalisme ordinaire?

8 De fait, la question de l'expulsion paraît d'emblée s'inscrire au croisement du débat sur le statut des étrangers et de la question de la réforme pénale. Comme le rappelle Rogers Brubaker, la possibilité d'expulser les étrangers, les nationaux étant définitivement protégéscontre une telle mesure, est une technique cruciale de « clôture du territoire $»^{15}$. Le dispositif répressif des Etats modernes parachève cette fermeture en prévoyant en général une peine d'emprisonnement pour les contrevenants qui se rendraient coupables du délit de "rupture de ban », c'est-à-dire qui demeureraient malgré tout sur le territoire.

9 Dans le cas français, ce couple expulsion-prison tel qu'il existe dans les années 1930 est un héritage ancien : celui de la loi du 3 décembre 1849. Elle prévoit simplement que le ministre de l'Intérieur peut prononcer l'expulsion de tout étranger séjournant ou voyageant sur le territoire, et le faire conduire à la frontière. L'article qui suit précise que l'étranger qui se sera soustrait à la mesure d'expulsion « ou qui, après être sorti de France, y aura pénétré de nouveau sans autorisation", sera condamné à un emprisonnement de un à six mois ${ }^{16}$.

10 La notion d'éloignement effectif n'est donc pas absolument nouvelle dans les années 1930. Pour autant, la reconduite à la frontière et la rétention administrative sont tombées en désuétude au cours du XIXème siècle. A l'époque où nous nous plaçons, la pratique en la matière consiste seulement à notifier l'arrêté d'expulsion à l'étranger, lequel doit par la suite quitter le territoire par ses propres moyens. En aval de cette notification, il n'existe pas de dispositif pour assurer effectivement son départ - ce qui ne pose alors aucun problème réel. L'association entre le prononcé de l'expulsion et le travail de "redressement des volontés » assuré par la peine de prison est perçue comme suffisante à garantir le départ du territoire et sa "clôture ». Un juriste peut encore dire à ce propos en 1933 que « la prison est encore le meilleur moyen pour obliger les hommes de mauvaise volonté à payer et à faire ce qu'ils doivent $»^{17}$.

11 C'est dans cette situation de "vide juridique » relatif qu'est réactivée la thématique de l'éloignement effectif des étrangers, autour des expulsés «inexpulsables » : ceux qui demeurent malgré tout dans leur pays d'accueil et subissent, le plus souvent malgré eux, des condamnations à répétition pour infraction à un arrêté d'expulsion. On parlera à leur propos de "non-délit impossible ", ce qui n'empêche pas les magistrats de prononcer les condamnations sans tenir compte de la situation particulière des accusés ${ }^{18}$.

12 De même, les représentations dominantes de l'époque les associent moins à la nouveauté de cette situation qu'aux classiques représentations des «pauvres dangereux » : 
13 «Dans ces conditions, on expulse, mais sans réaliser l'expulsion. On expulse même lorsque l'expulsion est pratiquement irréalisable (...). N'ayant pu obtempérer, ils sont traduits devant les tribunaux, condamnés et emprisonnés. A peine libérés(...) ils sont appréhendés et condamnés derechef. (...) On crée ainsi des récidivistes qui encombrent les tribunaux et les administrations et qui parfois se déclassent peu à peu socialement, risquant de devenir véritablement dangereux pour la sécurité publique $»^{19}$.

14 «(..) Ces étrangers ne deviennent-ils pas beaucoup plus nocifs qu'auparavant (...). Subissant dans les maisons d'arrêt la contagion des malfaiteurs professionnels, ils viendront à leur tour grossir l'armée du crime, et risqueront, quelque jour, de devenir un ferment révolutionnaire ${ }^{20}$.

Les étrangers en rupture de ban sont avant tout ici une sous-catégorie de la population pénale en général, quelle que soit sa nationalité. A l'origine, ils s'installent dans une situation, littéralement, de transit qui n'avait théoriquement aucune vocation à durer. Comme dans le cas des chômeurs étudiés par E. Pierru, c'est la stabilisation dans cette position durable de marginalité, avant même l'examen de la cause de cette situation, qui tire les expulsés inexpulsables vers la thématique plus générale des illégalismes à placer sous surveillance ${ }^{21}$. Dans le premier extrait que nous citons, la durée devient directement criminogène. L'inexpulsable croise cette fois-ci la figure du récidiviste, et, comme au XIXème siècle, l'idée d'un caractère criminel (qui fait de l'étranger un élément "véritablement dangereux») relié non au délit ou à la série de délits initialement commis, mais au fait même de la répétition du crime et à l'installation dans une « criminalité d'habitude $»^{22}$. L'étranger ici s'installe dans un cycle infernal où, ayant perdu ses ressources et finalement jusqu'aux caractéristiques de la civilisation, il doit en outre repasser nécessairement par la prison, dès lors qu'il ne lui est plus possible de quitter la France et de se soustraire ainsi aux sanctions de la loi de 1849 .

Le jugement porté sur les inexpusables prolonge donc les représentations disciplinaires des foules délinquantes héritées du XIXème siècle. Il les renvoie à la thématique du déclassement et de la dé-civilisation, mais aussi à la connexion entre criminalité et agitation politique, entre atteinte à l'ordre et violence révolutionnaire. Comme chez Foucault, la gestion de l'étranger inexpulsable traduira alors la volonté de contrôler «(...) [le] grouillement imprécis [de] ces troupes incertaines de vagabonds qui recrutent selon leur passage et les circonstances, des chômeurs, des mendiants, des réfractaires, et qui se gonflent parfois (...) jusqu'à former des forces redoutables de pillage et d'émeute ", pour lui substituer « un groupe relativement restreint et clos d'individus sur lesquels on peut exercer une surveillance constante $" 23$.

Repenser l'économie de l'expulsion

17 Ces étrangers dont le danger est évoqué dans les termes propres à tous les « illégalismes populaires », vont pourtant devoir être isolés lorsqu'il s'agira d'élaborer l'agencement institutionnel censé les gérer et assurer leur surveillance. Les acteurs du débat de l'entre-deux-guerres s'accordent en effet pour constater l'échec du couple classique reliant l'expulsion à l'emprisonnement pour rupture de ban. Là où l'incarcération reste chez Foucault le moyen essentiel de redressement du délinquant en dépit du constat renouvelé de son échec, la logique de l'articulation entre expulsion et prison, dans le cas bien spécifique des étrangers, ne tient plus. Comme tout illégalisme, cette masse indistincte d'expulsés que l'on ne parvient pas à éloigner matériellement du territoire devra pourtant elle aussi être saisie, quadrillée, divisée en catégories et en classes bien particulières ${ }^{24}$. Mais il ne pourra alors s'agir que de nouveaux dispositifs juridiques différenciés, destinés à gérer de façon spécifique les 
différentes situations des "expulsés inexpulsables ", en les faisant ainsi émerger comme catégories distinctes de la foule « ordinaire » des populations criminelles. Ceci ne signifie en aucun cas la rupture avec le «diagramme disciplinaire» : sa logique immanente reste la même. Mais, pour saisir et gérer efficacement cette population désormais entrée dans un espace de "visibilité » et devenue objet d'une série d'énoncés, il doit être réorganisé dans ses relais et ses points d'exercice ${ }^{25}$. Nous allons ici l'examiner à travers deux catégories d'inexpulsables, telles que les acteurs les construisent eux-mêmes, et telles qu'elles se mettent à exister à travers l'invention, à leur intention, de nouveaux modes de gestion et de surveillance.

Les « sans le sou » à reconduire

18 La première catégorie isolée par les différents intervenants est celle que R. Fatou, substitut du Procureur d'Aix-en-Provence, qualifie à plusieurs reprises de "sans le sou »: c'est-à-dire la masse des étrangers qui restent en France faute de pouvoir financer le voyage de retour, et sont donc contraints financièrement au non-respect de l'arrêté d'expulsion.

19 Ce qui n'est apparemment qu'un problème financier place en effet la gestion carcérale des étrangers inexpulsables devant une limite: sanctionner un délit qui reste involontaire. Le couple expulsion-emprisonnement se heurte ici aux résultats d'une évolution juridique bien postérieure à la loi de 1849: la construction progressive, décrite par G. Noiriel, d'un statut de l'étranger, objectivé dans un ensemble de catégories juridiques ${ }^{26}$. Le défaut de ressources n'est pas en effet lié à la seule pauvreté des étrangers frappés d'expulsion. Il est également organisé par la gestion étatique de la main-d'oeuvre étrangère depuis que le protectionnisme, et particulièrement la protection du marché du travail national, est devenu la grande affaire des Etats-Nations européens ${ }^{27}$. Lorsque l'étranger "émerge » au XIXème comme catégorie juridique objectivée, c'est à travers le problème de son poids économique, notamment parce qu'il occupe les emplois des nationaux - vision de l'étranger que la crise, on l'a dit, ne fait qu'accentuer.

20 Le «sans ressources" visé ici, c'est donc avant tout le représentant de cette immigration de travail polonaise ou italienne dont il était question en introduction. Quant au défaut de ressources qui l'oblige à rester sur le territoire, il est « organisé » par les effets imprévus du document le plus représentatif du souci "protectionniste » de l'Etat: la carte d'identité d'étranger, équivalent du permis de séjour valant également permis de travail. Elle permet en effet l'expulsion à motif économique : retirer sa carte à un étranger occupant un emploi dans un secteur touché par le chômage, c'est le priver de son travail, mais aussi lui signifier un refus de séjour dont le non respect peut entraîner l'expulsion ${ }^{28}$. Mais l'expulsion elle-même entraîne logiquement le retrait de la carte :

21 "A ceux qui, sortant de prison les poches vides, car en prison le travail est inexistant et peu rémunérateur, on serait tenté de dire: "gagnez par votre travail l'argent nécessaire à votre exode!» Mais voilà que par le jeu même de l'arrêté d'expulsion, leur carte d'identité leur est retirée, et dès lors, impossible pour eux de s'embaucher: le patron qui, même par pitié, les prendrait à son service, s'exposerait aux pénalités du livre II du code du travail! Ces malheureux semblent donc enfermés dans un véritable cercle infernal $»^{29}$.

22 «Et c'est ainsi que la plupart des expulsés demeurent volontairement ou involontairement en France, y subissent condamnations sur condamnations pour infraction à l'arrêté d'expulsion qui les frappe (...) car il ne faut jamais perdre de vue que la simple notification d'un arrêté 
d'expulsion, non suivie d'une exécution effective, entrave l'oeuvre d'assimilation et aboutit en fin de compte à priver l'étranger, obligé de vivre de son travail, des pièces réglementaires qui lui seraient nécessaires, ne serait-ce que pour gagner l'argent qui lui permettra d'assurer son départ. (...) Privés du droit au travail ne sont-ils pas amenés à rechercher leur subsistance dans le vol? $»^{30}$.

C'est donc autour de la nouvelle question du travail que la décision d'éloignement crée en quelque sorte les conditions de sa propre inefficacité. L'inexpulsable renvoie plus que jamais à cet "oisif irrécupérable » et en cours de déclassement dont on a déjà évoqué la perception en termes disciplinaires : le retrait de la carte le place au chômage forcé, lui interdit ensuite de quitter le territoire; sa persistance sur le territoire transforme alors le chômage forcé en chômage permanent, lui interdisant de « s'assimiler » en gagnant sa vie en France. Par le jeu de la carte d'identité, le chômeur étranger n'est donc plus seulement un poids économique. Il est plus généralement placé dans un continuum reliant la situation irrégulière, l'absence d'emploi régulier et le crime -vol ou vagabondage - dans un glissement progressif vers la marginalité ${ }^{31}$. Dans cette situation, la discontinuité expulsion - retrait de la carte d'identité - chômage forcé - prison constitue un cercle vicieux, à l'opposé de ce que serait une économie continue des peines et une gestion cohérente de l'éloignement. Le contrôle de l'expulsé ne peut alors se limiter à la peine de prison pour rupture de ban, qui paraît ici inutile et incongrue. Dans sa logique de persuasion et de redressement du délinquant, elle est durablement déconnectée de la gestion d'une catégorie de délinquants pour lesquels le discours du redressement des volontés n'est plus tenable.

Il s'agit, dès lors, de remettre en vigueur la vieille exigence de la loi de 1849 : celle de la reconduite à la frontière, désormais nécessaire pour "reconstruire " une gestion cohérente de la foule des inexpulsables ${ }^{32}$. Réexaminée au début des années 1930, la mesure prend toutefois un sens nouveau. Puisqu'elle se destine à un "sans ressources » également perçu comme un criminel déclassé, elle sera volontiers reformulée dans les termes de la philanthropie du XIXème siècle : une volonté d'assistance mêlée au désir de surveiller la population aidée, pour en contrôler au plus près la déviance. De façon significative, c'est à la faveur d'un Congrès national des associations de patronage ${ }^{33}$ que quelques-uns des acteurs que nous avons évoqués résument dès 1933 la problématique. Si la mesure de reconduite est détachée de l'emprisonnement, elle reste donc conçue comme une extension de la logique du « caritatif-disciplinaire » :

"Se borner à payer le voyage d'un étranger expulsé, c'est sans doute un petit acte de charité (...) : ce n'est pas du patronage, car l'oeuvre du patronage s'exerce en profondeur, par une action lente et continue. Ce qu'il faut désirer, c'est qu'à l'assistance purement matérielle s'ajoute un commencement d'assistance morale, c'est que le patronage de France, qui a rapatrié l'expulsé libéré, puisse le recommander à un patronage de son pays $»^{34}$.

Avant d'être la mesure répressive qu'elle est largement devenue aujourd'hui, la reconduite à la frontière est donc ici envisagée comme une partie de l'oeuvre plus générale de relèvement des condamnés libérés, en quoi consiste exactement le "patronage». L'aspect financier du traitement assistanciel de la pauvreté doit s'accompagner d'une moralisation, et d'un contrôle. Cette alliance entre la gestion d'un poids économique inutile et la répression de la déviance éventuelle, transparait également dans la volonté de pister les fraudeurs potentiels; non pas ceux qui chercheraient à éviter la reconduite, mais ceux qui voudraient abuser d'un dispositif décidément perçu comme une aide : 
27 «Combien coûterait le soi-disant rapatriement de tous ceux que leurs affaires ou leur plaisir appellent en France, et qui seraient assurés d'un billet de retour gratuit, sous la forme d'un arrêté d'expulsion qu'il sera toujours facile de mériter et qui ne comportera plus aucune sanction pénale? $»^{35}$.

28 La disparition de la «sanction pénale " laisse le discours des réformateurs face à la seule thématique du "passager clandestin » abusant indûment des aides qui lui sont proposées. Elle justifiera par la suite l'accentuation de la surveillance afin de distinguer les étrangers "vraiment nécessiteux » de ceux qui useraient de la reconduite comme d'un moyen commode de franchir les frontières. Mais il est d'autres cas dans lesquels la logique répressive pourra plus directement s'exprimer.

Le sans-Etat, ou comment s'en débarrasser

29 La reconduite à la frontière pour les étrangers nécessiteux n'est en effet pas la seule mesure rendue nécessaire par les mutations de la législation nationale ou internationale, et leurs effets sur les migrations. Le mouvement vers un contrôle étatique des déplacements est à l'origine d'une autre évolution juridique, qui remet également en cause le couple traditionnel expulsion-prison : l'invention du passeport et $\mathrm{du}$ visa pour l'entrée sur le territoire d'un Etat étranger. ${ }^{36}$ Cette exigence nouvelle, véritablement instituée après la première guerre mondiale, pose un problème dès lors que se multiplient les cas d'étrangers apatrides, privés de leur nationalité par simple effet de la loi de leur pays d'origine, ou par sanction politique dans le cas des réfugiés : ce sont ces étrangers qu'Arendt nommait les « sans-Etat ", et ce sont eux qui posent à la logique de la loi de 1849 un nouveau problème. Là aussi, l'apatride expulsé reste sur le territoire, mais cette fois faute de pouvoir être accepté par aucun Etat, y compris par son Etat d'origine qui ne le reconnaît plus - c'est-à-dire, faute de pouvoir se faire délivrer un passeport national et un visa l'autorisant à voyager ${ }^{37}$.

Dans ce cas comme dans le précédent, la prison n'est plus à même de s'insérer dans un ensemble reliant la clôture durable du territoire par l'expulsion, et cette autre clôture, à l'entrée du territoire étranger cette fois, que constitue l'obligation du passeport :

31 «(...) la pratique judiciaire [consistant à condamner l'étranger pour être resté sur le territoire alors qu'il n'a aucun moyen de sortir] accumule sur la tête d'un malheureux infracteur (...) récidiviste malgré lui, victime des circonstances beaucoup plus que de sa faute, un nombre de mois et d'années de prison sans rapport avec la gravité du délit originaire qui peut lui être reproché $»^{38}$.

32 Là encore, la gestion strictement carcérale de ce qui apparaît comme un délit forcé ne parvient pas à trouver de justification. Là encore, son effet est contraire à l'objectif de gestion disciplinaire et de surveillance de la délinquance étrangère, puisqu'elle contribue à pérenniser la situation de transit de l'étranger. Il est donc également nécessaire ici de remplacer le couple expulsion-emprisonnement par une nouvelle architecture juridique, plus adaptée.

33 Pour autant ce cas est à distinguer de la situation précédente des "sans le sou », bien qu'il participe de la même problématique. Non que le problème de leur poids économique ne soit pas envisagé : leur accès au marché du travail peut poser problème, en raison de la concurrence qu'ils suscitent pour les emplois les plus qualifiés ${ }^{39}$. Mais s'ils sont envisagés, à l'instar des autres étrangers, comme des travailleurs indésirables, leur situation d'inexpulsables "définitifs", pour des raisons juridiques et non plus matérielles, interdit d'aborder la question de leur coût pour l'économie française dans 
l'optique de la reconduite à la frontière et du financement du retour. Si l'emprisonnement doit disparaître, il doit être remplacé par un agencement incluant d'autres mesures. Or ces mesures sont évoquées dans une arène infiniment plus vaste que le simple débat entre pénalistes nationaux. Les apatrides et réfugiés dépourvus de nationalité et de passeport, toujours plus nombreux après 1933, constituent en effet un problème en soi, traité à l'échelon international. La Société des Nations multiplie les " arrangements » à leur propos, tandis que la VIIème conférence internationale pour l'unification du droit pénal réunie début 1938 charge l'une de ses commissions d'étudier spécifiquement la question de leur expulsion. Tout comme les textes de la SDN, la déclaration finale admet que "lorsque l'exécution de l'expulsion n'est pas réalisable, il pourra y être substitué des mesures de sûreté ou de surveillance» - ce qui renvoie potentiellement à l'internement. Plus généralement, la question des camps est thématisée dès le début des années 1930 par les spécialistes du droit international comme une solution potentielle au problème des réfugiés. Au cours des années 1930, bon nombre d'Etats, tous régimes confondus, ouvrent des camps pour assurer la surveillance des sans-Etat inexpulsables. Dans son rapport devant le Comité français de droit international privé, H. Donnedieu de Vabres peut ainsi énumérer les expériences « internementales » de la Belgique, de l'Estonie ou encore de la Yougoslavie ${ }^{40}$.

$\mathrm{Au}$ sein du débat français, le camp est une fois de plus envisagé dans les termes de la défense sociale. Il s'insère cependant dans un nouvel agencement du contrôle des expulsés, adapté à la gestion des sans-Etat, mais qui traduit aussi un déplacement de la surveillance.

35 Ce qui apparaît tout d'abord en effet, en amont même de l'effectuation matérielle des expulsions, c'est la volonté d'en limiter le nombre en en encadrant la pratique. Cet aspect mériterait un développement plus conséquent. Disons seulement ici qu'il s'agit de compenser l'arbitraire de l'expulsion administrative en confiant l'expulsion au juge pénal exclusivement ${ }^{41}$. D'autre part, l'expulsion doit être impossible pour certaines catégories d'étrangers, que plusieurs projets de réformes se proposent de définir ${ }^{42}$.

Ce n'est qu'à la condition d'avoir effectué ce tri que les « mesures de sûreté " peuvent être envisagées : elles ne doivent alors viser que les étrangers qui, passés au crible des "catégories protégées", constituent pour H. Donnedieu de Vabres les "étrangers dangereux » - en quelque sorte, les seuls qui soient suffisamment "irrécupérables » pour que l'argument du délit involontaire puisse être surmonté. On y trouve donc les inexpulsables coupables de crimes graves, mais également un motif important d'expulsion, qui n'avait pas été évoqué jusqu'ici : la perception d'une menace politique liée spécifiquement aux réfugiés, qui ne fait qu'augmenter à mesure que la perspective d'un conflit se rapproche en Europe ${ }^{43}$. C'est pour cette catégorie d'expulsés inexpulsables que sera prévu "un système de mesures de sûreté, destinées à remplacer les courtes peines d'emprisonnement, dont le procès n'est plus à faire ${ }^{44}$.

Le camp est au nombre de ces mesures qui se substituent à la prison pour la gestion des étrangers inexpulsables. Toutefois, il se relie à une série d'institutions avec lesquelles il interagit. Pour ces étrangers «irréductiblement» dangereux en effet, on prévoit «la résidence obligatoire dans une localité assignée(...) l'institution de camps de travail [et] en désespoir de cause, la relégation facultative $»^{45}$.

38 A travers la référence au travail, le camp reste une manière de gérer le poids économique des étrangers inexpulsables en conférant une utilité à des internés aisément perçus comme des « bouches inutiles $»^{46}$. Mais la référence au travail - forcé - 
renvoie également à l'autre mesure évoquée ici, celle de la relégation. Cette dernière peine, qui suppose l'envoi du condamné vers les bagnes coloniaux, est lourde, là encore, de l'héritage de la défense sociale du XIXème siècle. Elle renvoie à « une philosophie pénale qui n'est plus axée seulement sur l'emprisonnement [et qui] met au coeur de sa dynamique : l'exclusion $»^{47}$.

Cette dynamique d'exclusion, le camp en est désormais un élément clé : camp de travail, dans lequel la main d'oeuvre des étrangers indésirables pourrait être utilement employée, il se mue éventuellement en " camp de colonisation agricole ${ }^{48}$, alliant à la fois la «réhabilitation par le travail» et ce que l'on n'hésite pas à nommer une "mesure de débarras $»^{49}$. Jusque dans le commentaire final du décret-loi du 12 novembre 1938, on estime que "l'intervention éventuelle du ministre des colonies laisse supposer que certains de ces centres seront peut-être établis en Afrique. La surveillance devant être permanente laisse à entendre que ces centres seront peut-être... des camps de concentration $»^{50}$.

40 La figure du camp, notamment par l'intermédiaire du travail forcé, interagit donc avec une logique d'exclusion pure et simple de "l'inexpulsable - irrécupérable » hors du territoire national. Ce trait est d'autant plus notable qu'à la même époque, le discours pénal d'exclusion dont parle M. Kaluszynski perd de sa force dans le cas des nationaux : alors que cette thématique est encore soutenable pour les apatrides inexpulsables, et à propos même des Centres spéciaux de rassemblement de novembre 1938, il a cédé la place dans le cas des criminels nationaux à un discours abolitionniste, qui débouche au contraire sur la fermeture des bagnes par un décret du 17 juin de la même année ${ }^{51}$.

41 Le premier interné des camps pour étrangers «croise» en quelque sorte le dernier forçat du bagne. Si les deux figures sont réunies par le discours du « débarras » dont elles font toutes deux l'objet, ce passage de témoin peut également traduire un déplacement dans la cartographie générale du pouvoir. Comme si l'inexpulsable étranger, promis au camp-bagne colonial, constituait le reliquat d'une représentation plus ancienne du criminel en passe de disparaître pour les nationaux : c'est alors vers lui et vers le camp d'internement que se "déplacent" certains discours répressifs hérités du XIXème siècle, que l'on applique de moins en moins, à l'époque, aux délinquants français. On serait alors tenté de transposer sur la gestion de la population pénale la remarque de G. Noiriel, selon laquelle les catégories sociales ne bénéficiant pas d'une représentation dans l'espace public sont les dernières dont la gestion puisse encore relever de la police pure et simple, et non du champ politique ${ }^{52}$. Alors que les ouvriers, puis les femmes, ont progressivement bénéficié au XXème siècle d'une intégration au système politique, ainsi que de l'émergence de représentants propres et reconnus par l'Etat, les étrangers - peut-être de concert avec les chômeurs évoqués par E. Pierru - feront alors partie des catégories restées à l'écart, pour lesquelles le discours disciplinaire reste par conséquent acceptable. En ce sens, le camp autour duquel se réorganise le contrôle des étrangers expulsés est le révélateur - et l'opérateur - d'une nouvelle division entre nationaux et étrangers, cette fois-ci dans le champ pénal. 


\section{NOTES}

1. . Deleuze G., Foucault, Paris, Minuit, 1986, 141 p.

2. . Notamment par le vote de la loi du 10 août 1932 autorisant le gouvernement à restreindre par décret la proportion d'étrangers dans certaines branches de l'industrie et du commerce.

3. . Pour une présentation générale, voir Noiriel G., Le creuset français. Histoire de l'immigration, Paris, Le Seuil, 1988, 437 p. ; Noiriel G., Les origines républicaines de Vichy, Paris, Hachette, 1999, pp. 138 et suivantes.

4. . Sur ce point, voir Lewis M.D., « The Strangeness of Foreigners. Policing Migration and Nation in Interwar Marseille », French Politics, Culture and Society, vol. 20, n³, automne 2002, pp. 65-85 ; Lewis M.D., « Les pratiques d'expulsion dans le Rhône durant la crise ", in Rygiel P. (dir.), Le bon grain et l'ivraie. L'Etat-nation et les populations immigrées fin XIXème siècle - début XXème siècle, Paris, Editions Rue d'ULM/Presses de l'ENS, 2004, pp. 152-163.

5. . Arouni R., « Le débat autour du statut des étrangers dans les années 1930 », Le Mouvement Social, $\mathrm{n}^{\circ}$ 188, juillet-septembre 1999, pp. 61-75.

6. . Voir les actes du Congrès National des Sociétés de Patronage de 1933, Revue pénitentiaire et de droit pénal, nº-9-10, août-octobre 1933, pp. 335-373.

7. . Société savante fondée en 1935. La Commission des Etudes Législatives consacre également plusieurs séances à la question en 1938.

8. . Kaluszynski M., « Construire la loi. La Société Générale des prisons (1877-1900) », in Kaluszynski M., Wahnich S. (dir.), L'Etat contre la politique? Les expressions historiques de l'étatisation, Paris, L'Harmattan, 1998, pp. 205-221. Parmi les « nouveautés », on trouvera notamment les propositions de loi Moutet en 1934 ou celle de X. Vallat en 1937, qui reprennent explicitement les propositions des sociétés savantes.

9. . Callon M., "Eléments pour une sociologie de la traduction ", Année sociologique, $\mathrm{n}^{\circ} 36,1986, \mathrm{pp} .170-206$.

10. . Il s'agit dans les deux cas de « spécialistes » reconnus à l'époque; l'un démographe et sociologue, l'autre haut fonctionnaire et chef du Service de la main d'oeuvre et de l'immigration agricoles.

11. . J.P. Niboyet est Secrétaire Général du Comité. Voir Noiriel G., Les origines républicaines de Vichy, op. cit., pp. 135-137.

12. . Débat doctrinal qui accompagne la IIIème République. Sur ce point, voir Kaluszynski M., op. cit.

13. . Notamment Raymond Fatou, Substitut du Procureur Général à Aix en Provence, fréquemment invité pour évoquer le problème.

14. . Premier président de la Cour de Cassation, Paul Matter est également Président d'Honneur du Comité Français de Droit International Privé, et co-auteur au même moment d'un projet de réforme du Code Pénal généralisant l'expulsion des délinquants étrangers. Sur ce point, voir Lascoumes P., Poncela P., Lenoël P., Les grandes phases d'incrimination, Paris, Ministère de la Justice, 1992, pp. 72-76.

15. . Brubaker R., Citoyenneté et nationalité en France et en Allemagne, Paris, Belin, 1997, $319 \mathrm{p}$.

16. . Loi du 3 décembre 1849, Bulletin des lois, 11 décembre 1849. 
17. . Intervention de Raoul Pascalis, fonctionnaire à la Préfecture de police de Paris et Secrétaire général de l'Union des Sociétés de Patronage de France, Revue pénitentiaire et de droit pénal, op. cit., p. 369.

18. . Jusqu'en 1939, en effet, la jurisprudence ne reconnaît ni l'argument de « force majeure ", ni celui de l'irresponsabilité qui sont pourtant fréquemment soulevés par les avocats.

19. . Intervention de Marcel Paon, séance du 8 avril 1935, Travaux du Comité français de droit international privé, n¹-2, Paris, Dalloz, 1934-35, p. 115.

20. . Fatou R., « Note sur le problème des étrangers en France, et spécialement sur l'inefficacité, les inconvénients et les dangers des mesures d'expulsions non suivies d'exécution effective ", Revue pénitentiaire et de droit pénal, op. cit., p. 341.

21. . Pierru E., « La politique des ventres creux. Stigmatisations socio-politiques du chômeur dans la grande dépression. Jalons pour une histoire comparée ", Communication à la table ronde « La radicalisation politique ", VIIème congrès de l'AFSP, Lille, septembre 2002.

22. . Kaluszynski M., « Le criminel à la fin du XIXème siècle : un paradoxe républicain ", in Gueslin A., Kalifa D., Les exclus en Europe 1830-1930, Paris, Les Editions de l'Atelier, 1999, pp. 253-265.

23. . Foucault M., Surveiller et punir. Naissance de la prison, Paris, Gallimard, 1975, p. 324.

24. . Le schéma est identique à celui de la réforme de la prison chez Foucault : il s'agit moins d'assurer réellement le départ des expulsés que de « saisir » et réguler une population qui échappait jusqu'ici à tout contrôle. De fait, de 1933 à 1939, les différentes « innovations » législatives ne parviennent pas à clore le débat sur l'éloignement effectif. Pour le discours équivalent sur l'expulsion contemporaine, voir Bietlot M., « Du disciplinaire au sécuritaire : de la prison au centre fermé », Multitudes, $\mathrm{n}^{\circ} 11$, hiver 2003, pp. 57-66.

25. . Deleuze G., Foucault, op. cit.

26. . Noiriel G., La tyrannie du national, Paris, Calmann Lévy, 1991, 355 p.

27. . Noiriel G., « Représentations nationales et catégories sociales. L'exemple des réfugiés politiques ", in Etat, nation et immigration. Vers une histoire du pouvoir, Paris, Belin, 2001, pp. 259-287.

28. . Il s'agit de la pratique connue à l'époque sous le nom de « refoulement ». A ce sujet, voir Rygiel P., « Refoulement et renouvellement des cartes de « travailleur étranger » dans le Cher durant les années 30 », in Rygiel P. (dir.), Le bon grain et l'ivraie, op. cit., pp. 117-133.

29. . Fatou R., « Note sur le problème des étrangers en France, ... » op. cit., p. 338.

30. . Fatou R., «A propos du problème des étrangers indésirables et de leur expulsion non suivie d'exécution effective ", Revue de Science Criminelle et de droit pénal comparé, t.1, 1936, pp. 317-330.

31. . Pour une analyse contemporaine de ce discours, voir Quassoli F., « The Judicial System and the Social Construction of Migrants's Criminality : the Case of Milan », in Body-Gendrot S., Martiniello M., Minorities in European Cities. The dynamics of Social Integration and Social Exclusion at the Neighbourhood Level, London, MacMillan, 2000, pp. 203-216.

32. . De fait, elle est instituée par la circulaire du 4 décembre 1934 - mais avec des moyens très faibles.

33. . Le patronage désigne à l'époque l'assistance/surveillance des détenus libérés. Voir Kaluszynski M., Production de la loi et genèse des politiques pénales. La Société Générale des 
prisons 1877-1900, Rapport terminal remis au Ministère de la Justice, CERAT, octobre 1996, pp. 117-131.

34. . Intervention de Raoul Pascalis au Congrès national des Associations de patronage, Revue pénitentiaire et de droit pénal, 1933, op. cit., pp. 371-372. Les débats de ce congrès, consacrés à l'expulsion, voient également intervenir Paul Matter et Raymond Fatou. 35. Ibid., p. 371.

36. . Torpey J., The Invention of the Passport. Surveillance, Citizenship and the State, Cambridge, Cambridge UP, 2000, $211 \mathrm{p}$.

37. . Arendt H., L'impérialisme, Paris, Seuil, 1997, 348 p.

38. . Rapport de Henri Donnedieu de Vabres, Travaux du Comité français de droit international privé, op. cit., Séance du 26 février 1935, p. 53.

39. . C'est notamment à leur encontre qu'est adoptée à partir de 1933 une législation restreignant le nombre des étrangers autorisés à exercer les professions de médecin et d'avocat. Voir Noiriel G., Les origines républicaines de Vichy, op. cit., pp. 141 et suivantes. ; Weil P., Qu'est-ce qu'un Français? Histoire de la nationalité française depuis la Révolution, Paris, Grasset, 2002, pp. 88-89.

40. . Ibid., pp. 77-78. Sur la problématisation internationale de l'internement, voir Philonenko M., « Expulsion des Heimatlos », Journal du droit international, n6, novembre-décembre 1933, pp. 1161-1187.

41. . L'idée est également présente dans le « projet Matter » de réforme du code pénal, voir supra.

42. . Il s'agit notamment des étrangers mariés à des Françaises, des mineurs ou encore des étrangers ayant servi dans la Légion étrangère. Voir supra les propositions de loi Moutet et Vallat.

43. . Sur ce point, voir Badia G., Les barbelés de l'exil. Etudes sur l'immigration allemande et autrichienne 1938-1940, Grenoble, PUG, 1979, 443 p.

44. . Donnedieu de Vabres H., Travaux du Comité français de droit international privé, op. cit., p. 61 .

45. . Ibid, p. 61.

46. . Bernardot M., « Le pays aux mille et un camps. Approche socio-historique des espaces d'internement en France au XXème siècle ", Cahiers du Cériem, $\mathrm{n}^{\circ}$ 10, décembre 2002, pp. 57-76.

47. . Kaluszynski M., " Le criminel à la fin du XIXème siècle... », op. cit., p. 254.

48. . Intervention de P. Matter, Revue pénitentiaire et de droit pénal, n8-9-10, aoûtoctobre 1933, p. 351.

49. . Donnedieu de Vabres H., Travaux du Comité français de droit international privé, séance du 8 avril 1935, op. cit., p. 98.

50. . Morice B., « Commentaire du décret-loi du 12 novembre 1938 relatif à la situation et à la police des étrangers ", Les lois nouvelles, 1939, p. 80.

51. . Petit J.G (dir.), Histoire des galères, bagnes et prisons, Toulouse, Privat, 1991, 368 p.

52. . Noiriel G., « Représentations nationales et catégories sociales... », op. cit. 
INDEX

Mots-clés : étrangers, Exilés, expulsions, histoire

Index géographique : France

Index chronologique : 1930 - 1940 